\title{
Predictores biosocioculturales y estilos de vida de los estudiantes universitarios de una universidad de Huánuco.
}

\author{
Maria Luz Ortiz-Cruz ${ }^{1, a}$, Mely Meleni Ruiz-Aquino ${ }^{1, b}$
}

\section{RESUMEN}

Objetivos: determinar la relación entre los predictores biosocioculturales y los estilos de vida de los estudiantes universitarios. Material y métodos: investigación cuantitativa, de tipo transversal, analítico, prospectivo y observacional; con diseño correlacional. La población fue de 3261 estudiantes de la Universidad Privada de Huánuco, con una muestra de 343,7 estudiantes, seleccionados probabilísticamente por estratos, a quienes se les aplicó un cuestionario de predictores y una escala de estilos de vida, previamente validados. Se tuvo en cuenta los aspectos éticos de la investigación. Se realizó un análisis bivariado mediante la prueba Chi Cuadrado de Pearson, con $\mathrm{p} \leq 0,05$; apoyados en el PASW V20.0. Resultados: se comprobó la relación entre la condición de pertenecer al grupo adolescente $\left[\mathrm{X}^{2}=15,54 ; \mathrm{Gl} 3 \mathrm{y} \mathrm{p}=0.00\right]$ y la realización de trabajo paralelo al estudio $\left[\mathrm{X}^{2}=18,8\right.$; G1 3 y $\mathrm{p}=0.00]$ con el estilo de vida poco saludable. El pertenecer al género masculino $\left[\mathrm{X}^{2}=13,35 ; \mathrm{Gl} 3 \mathrm{y} \mathrm{p}=0.00\right]$ se relaciona con el estilo de vida saludable. No hubo relación entre la procedencia de la zona urbana $\left[\mathrm{X}^{2}=1,00 ; \mathrm{X}^{2}\right.$ $=13,35$; Gl 3 y p $=0.80]$, la tenencia de algún compromiso conyugal $\left[\mathrm{X}^{2}=13,35\right.$; GL 3 y $\left.\mathrm{p}=0,44\right]$, la tenencia de alguna religión $\left[\mathrm{X}^{2}=2,86\right.$; G1 3 y $\left.\mathrm{p}=0.41\right]$ y la condición de cursar más del VI ciclo académico $\left[\mathrm{X}^{2}=1,10\right.$; G1 3 y $\mathrm{p}=0.781]$ con el estilo de vida saludable. Conclusiones: los predictores biológicos están relacionados con los estilos de vida saludable y poco saludables de los estudiantes.

PALABRAS CLAVE: Predictores biológicos, sociales, culturales, estilos de vida, universitarios, promoción de la salud, salud pública.

\section{Predictors biosocioculturales and lifestyles of university student in an university of Huánuco.}

\section{SUMMARY}

Objectives: to determine the relationship between predictors and biosocioculturales lifestyles of college students. Material and Methods: quantitative research, transversal, analytical, prospective observational; with correlational design. The population was 3, 261 students of the Private University of Huanuco, with a sample of 343.7 students, probabilistically selected by strata, who were applied a questionnaire and a scale predictors lifestyles, previously validated. the ethical aspects of the research was taken into account. A bivariate analysis was performed by Chi Square test of Pearson, with $\mathrm{p} \leq 0.05$; supported by the PASW V20.0. Results: the relationship between the condition of belonging to the teen group [X2 $=15.54$ was found; G1 3 and $\mathrm{p}=0.00]$ and performing work parallel to the study $[\mathrm{X} 2=18.8 ; \mathrm{Gl} 3$ and $\mathrm{p}=0.00]$ with unhealthy life style. The male gender $[\mathrm{X} 2=13.35 ; \mathrm{Gl} 3$ and $\mathrm{p}=0.00]$ is related to healthy lifestyle. There was no relationship between the origin of the urban area $[\mathrm{X} 2=1.00 ; \mathrm{X} 2=13.35 ; \mathrm{Gl} 3$

Consejo Regional XII Huánuco, Colegio de enfermeros del Perú. Huanuco, Perú.

Doctora en enfermería; Decana

Magister en Salud Pública y Gestión Sanitaria 
and $\mathrm{p}=0.80]$, possession of a marital commitment $[\mathrm{X} 2=13.35$; GL 3 and $\mathrm{p}=0.44]$, possession of any religion [X2 $=2.86$; G1 3 and $\mathrm{p}=0.41]$ and the condition of pursuing academic year more than VI [X2 $=1.10$; G1 3 and $\mathrm{p}=0.781]$ with healthy lifestyle. Conclusions: biological predictors are related to healthy lifestyles and unhealthy students.

KEYWORDS: biological, social, cultural predictors, lifestyles, academics, health promotion, public health.

\section{INTRODUCCIÓN}

A nivel mundial, es preocupante el incremento de hábitos sedentarios, incluso en la población universitaria, con repercusión en el estado emocional y físico (1). Respecto a este último, una persona sedentaria tiene mayor riesgo de padecer múltiples enfermedades crónicas no transmisibles, como las cardiovasculares, hipertensión arterial, cáncer de colon, mama, endometrio, diabetes mellitus, y las osteomusculares que están relacionadas con el sobrepeso y la obesidad. Del mismo modo, es mayor la probabilidad de poseer niveles altos de colesterol y pérdida de densidad ósea. En cuanto al impacto emocional, se ha encontrado que una persona sedentaria tiene mayor probabilidad de padecer insomnio, depresión, ansiedad, estrés, entre otros; transformándose en determinantes de riesgo para el desarrollo de las enfermedades denominadas por la Organización Mundial de la Salud (OMS): las "enfermedades de los estilos de vida" (2).

Según la Organización de las Naciones Unidas (ONU) (3), los jóvenes se consideran como un grupo poblacional relativamente sano; sin embargo, algunos de sus problemas de salud, generalmente, se asocian a comportamientos relacionados con los estilos de vida. En este grupo etario, se hacen evidentes la ocurrencia de accidentes, el consumo de sustancias psicoactivas lícitas e ilícitas, las infecciones de transmisión sexual, los trastornos alimentarios y del sueño; entre otros. Aunque, la población universitaria representa un capital humano importante para la sociedad, las propias universidades y las políticas de salud carecen de estrategias concretas que favorezcan el estilo de vida saludable en esta población (4).

Se suman a dichas falencias las redes de apoyo familiar y las instituciones educativas influenciadas por su desempeño y exigencia académica, en una relación de causalidad circular que puede intensificar el riesgo. Prueba de ello, en Brasil, Lucas et al., (5), en el 2012, estimaron que la prevalencia del estilo de vida inadecuado fue de $5,3 \%$, donde los universitarios mayores de 20 años y con baja escolaridad materna ( $\leq$ de 9 años) presentaron un riesgo mayor para adquirir un estilo de vida inadecuado (5). A nivel del Perú, en Chimbote, Cotrina, Rodríguez
(6), en el 2014 , observaron que un $67,3 \%$ de los adultos jóvenes, tienden estilos de vida no saludables. En Trujillo, Gamarra, Rivera, Alcalde, Cabellos (7), 2010, mostraron una prevalencia de $23,5 \%$ de estilos de vida inadecuados en los estudiantes de enfermería.

En la ciudad de Huánuco, Ruiz et al., en el 2014 desarrollaron un estudio sobre "estilos de vida y estado nutricional de los estudiantes de la Universidad de Huánuco", donde observaron que alrededor de la mitad de la muestra tenían prácticas no saludables en lo que respecta a la actividad física, [52,9\%(182)] y en el autocuidado (8). Por su parte, Daza, en el 2014, encontró que un 7,2\% de los estudiantes universitarios tenían estilos de vida no saludables (9).

Una alternativa para reducir la vulnerabilidad a los riesgos de contraer múltiples enfermedades consiste en promover estilos de vida saludables, que en el mediano y largo plazo han demostrado éxito. Esto ha sido reportado en diferentes investigaciones y en casos particulares relacionados con el consumo de tabaco y alcohol, entre otros. Las intervenciones en segmentos de la población como los adolescentes y los jóvenes a la fecha no han evidenciado el impacto esperado para promover estilos de vida saludables y sostenibles (10). Departamento del Putumayo. [Tesis presentado como requisito de Especialización en Gerencia de la Salud Pública].

En tal efecto, el objetivo de la presente investigación, fue determinar la relación entre los predictores biosocioculturales y los estilos de vida de los estudiantes universitarios de Huánuco, priorizándose tal población, al no contarse con un análisis de los mencionados predictores que permitan el diseño de políticas e intervenciones de salud pública local, en el marco del modelo de universidad saludable.

\section{MATERIAL Y MÉTODOS}

El estudio fue de tipo observacional, prospectivo, analítico y transversal; con diseño correlacional y con enfoque cuantitativo, puesto que se probó las hipótesis mediante un análisis estadístico. La población fue de 
3261 estudiantes matriculados en el ciclo de verano de la Universidad de Huánuco, durante los meses de enero a marzo del 2014. La muestra lo conformaron 344 estudiantes, seleccionados por muestreo probabilístico estratificado según registro de matrícula, en la cual se consideró a 14 Escuelas Académicos Profesionales (Enfermería, Obstetricia, Odontología, Psicología, Ingeniería Civil, Ingeniería Ambiental, Ingeniería de Sistemas e Informática, Arquitectura, Derecho y Ciencias Políticas, Administración de Empresas, Contabilidad y Finanzas, Turismo, Hotelería y Gastronomía, Marketing Negocios Internacionales, Educación Básica: inicial y primaria).

Los datos se recolectaron a través de la encuesta autoadministrada, y los instrumentos aplicados fueron: un cuestionario de predictores y una escala de estilos de vida diseñado por Arrivillaga et al., en la versión de 68 reactivos, los que a través de un proceso de validación racional, delfos (mediante cinco expertos, quienes juzgaron la bondad de los reactivos), de aproximación a la población y de consistencia interna (confiabilidad) mediante el Alpha de Crombach ( $\mathrm{x}=$ $0,95)$, fueron modificados a 60 reactivos (11).

En la ejecución de la investigación se consideró los aspectos éticos (beneficencia, no maleficencia, autonomía y justicia); los cuales fueron plasmados en el consentimiento informado, firmado por la muestra en estudio.

En el análisis de los datos se aplicó la estadística descriptiva e inferencial, usando un $\mathrm{p}<0,05$ como el valor de la probabilidad del error en las pruebas de hipótesis, también, se consideró el análisis bivariado a nivel categórico entre todas las variables (las variables numéricas fueron categorizadas de acuerdo a estándares mundialmente conocidos y aprobados), con ello se aplicó la prueba no paramétrica de $\mathrm{X}^{2}$.

\section{RESULTADOS}

Respecto a las variables de la biología humana de los universitarios en estudio, se encontró, que cerca de la cuarta parte de ellos pertenecieron al grupo adolescente $[23,0 \%(79)]$ y cerca de la mitad [45,3\% (156)] fueron del género masculino. Sobre los aspectos sociales, la gran mayoría procedían de la zona urbana $[83,1 \%$ (286)], una mínima proporción de los estudiantes [7,0\% (24)] afirmó tener compromiso conyugal, y un total de $[34,9 \%(120)]$ tenía trabajo paralelo a sus estudios. Y en cuanto a los aspectos culturales, se apreció que la mayoría [88,4\% (304)] profesaban alguna religión; y alrededor de la cuarta parte $[26,2 \%(90)]$ pertenecían al VI ciclo de estudios.

Los estilo de vida, según la dimensión física o biológica, evidenció que un 52,3\% (180) de los estudiantes realizaban actividad física y ejercicio físico bajo supervisión entre ellos: caminatas, trotes, ejercicios de ayuda al funcionamiento del corazón (como aeróbicos, danza, baile, o practicaban taichí, yoga, meditación, relajación; entre otros). Además, terminaban el día con vitalidad y sin cansancio; sin embargo, por encima de la mitad de la muestra [64,2\% (221)] no mantenía estable su peso corporal ideal.

La participación de los estudiantes en actividades de recreación y manejo del tiempo libre fue en un 56,4\% (194), quienes practicaban caminatas, natación, ciclismo y además tenían momentos de descanso en su rutina diaria. Cabe resaltar que un $66,6 \%$ (229 estudiantes) no compartían con su familia y/o amigos el tiempo libre; tampoco realizaban actividades como ir al cine, leer, bailar, etc; y no destinaban parte de su tiempo libre para actividades académicas y/o laborales [59,0\% (203 estudiantes)].

El autocuidado y cuidado médico lo practicaban sólo un $45,6 \%$ (157 estudiantes); es decir, cerca de la mitad de ellos visitaron al médico y al odontólogo; controlaron su presión arterial, se practicaron exámenes de colesterol, triglicéridos, glicemia, de urología o ginecología (según su caso) durante los últimos seis meses y usaron bloqueadores al exponerse al sol. También, la mayoría evitó consumir ansiolíticos, tranquilizantes o estimulantes y la automedicación (en caso de dolores musculares, cefaleas o síndromes gripales). Casi en su totalidad, los estudiantes evitaron manejar bajo efectos del alcohol [96,5\% (332 estudiantes)], y no abordaron un vehículo manejado por algún conductor bajo efectos del licor u otras drogas; por último 56,1\% (193 estudiantes) usaron el cinturón de seguridad al conducir.

Por el contrario, alrededor de la mitad de los estudiantes [56,4\% (194 estudiantes)] no evitaban las exposiciones prolongadas a los rayos solares, no leían, ni seguían las instrucciones de los medicamentos cuando las utilizaba; no observaban su cuerpo con detenimiento para detectar cambios físicos; no obedecían las normas de tránsito, sea peatón o conductor y no tomaban en cuenta las señales de seguridad (prohibido fumar, peligro, a foro, etc.).

Por encima de la mitad de los estudiantes refirieron que sus hábitos alimenticios fueron saludables [59,6\% (205 estudiantes)], pues evitaban añadir sal a las comidas y 
azúcares a las bebidas en la mesa; no consumían más de 4 gaseosas a la semana, evitaban el consumo de dulces (golosinas), helados y pasteles más de dos veces a la semana, y limitaban el consumo de grasas (mantequilla, queso crema, carnes grasosas, mayonesa y salsas en general). Consumían carnes más de cuatro veces a la semana; limitaban su consumo de embutidos (jamón, salchichas, etc); mantenían un horario regular en las comidas, evitaban consumir comidas con contenido de ingredientes artificiales o químicos (colorantes y persevantes); y limitaban la ingesta de comidas rápidas [72,4\% (249 estudiantes)] (pizza, hamburguesa, salchipapa, etc.).

Por el contrario, una gran parte de los estudiantes [ $82 \%$ (282 estudiantes)] consumían productos ahumados; evitaban las dietas y los métodos que le prometían una rápida y fácil pérdida de peso. Llama la atención que alrededor de la mitad de la muestra en estudio, señaló no consumir entre cuatro y ocho vasos de agua al día $[51,2 \%$ (176 estudiantes)]; también, una importante mayoría no incluía alimentos balanceados en su dieta $[79,7 \%$ (274 estudiantes)]; casi las tres cuartas partes de la muestra no consumía pescado y pollo más que carnes rojas $[74,7 \%$ (257 estudiantes)]; en tanto, el 60,5\% (208 estudiantes) no desayunaba antes de iniciar su actividad diaria.

Más de la mitad de ellos, evitan el consumo de alcohol, tabaco y otras drogas [53,8\% (185 estudiantes)]; este hecho se expresaba en las respuestas de un "no" ante la propuesta de consumo de drogas; la evitación de fumar más de 2 cigarrillos al día, la prohibición de que otras personas fumen en su presencia; la limitación del consumo de licor al menos dos veces a la semana; el reconocimiento de cuándo empezar a beber y en qué momento parar; así mismo, el no consumo de drogas (marihuana, cocaína, bazuco, éxtasis, entre otras); de no más de dos tazas de café (al día) y de más de tres gaseosas en la semana. El sueño fue saludable en $66,0 \%$ (227 estudiantes) de los estudiantes ya que evitaban trasnocharse, se levantaban relajados luego de haber dormido, mantenían el sueño durante la noche, se daban una siesta, se mantenían lúcidos durante el día y evitaban el uso de pastillas para dormir. Sin embargo más de la mitad de los estudiantes refirió dormir al menos 7 horas diaria [59,9\% (206 estudiantes)].

La evaluación cualitativa del nivel del estilo de vida de los universitarios en estudio, determinó la categoría "saludable" en 51,5\% (177 estudiantes); un 29,1\% (100 estudiantes) se encontraban poco saludables; seguido de $12,5 \%$ (43 estudiantes) cuyo estilo de vida fue muy saludable; y por último una mínima proporción $[7,0 \%$ (24 estudiantes)] se hallaron no saludables (Figura 1).

$\mathrm{Al}$ analizar los predictores biosocioculturales en los estilos de vida de los estudiantes de una Universidad de Huánuco, se encontró relación significativa entre el predictor biológico: pertenecer al grupo de edad adolescente $\left(\mathrm{X}^{2}=\right.$ $15,54 ; \mathrm{Gl}, 3$ y $\mathrm{p}=0,00)$ y el estilo de vida poco saludable; con lo que se tuvo que rechazar la hipótesis nula. Esto expresa que la condición de adolescente representa un predictor del estilo de vida poco saludable de los universitarios en estudio (tabla 1).

Otro predictor biológico fue la condición de pertenecer al género masculino $\left(X^{2}=15,54 ; \mathrm{Gl}, 3\right.$ y $\left.\mathrm{p}=0.00\right)$ y los estilos de vida saludables de los estudiantes universitarios; por lo que se tuvo que rechazar la hipótesis nula, y se admite que los estudiantes masculinos tienen tendencia a desarrollar estilos de vida saludables (tabla 2).

\section{Condición del estilo de vida en general}

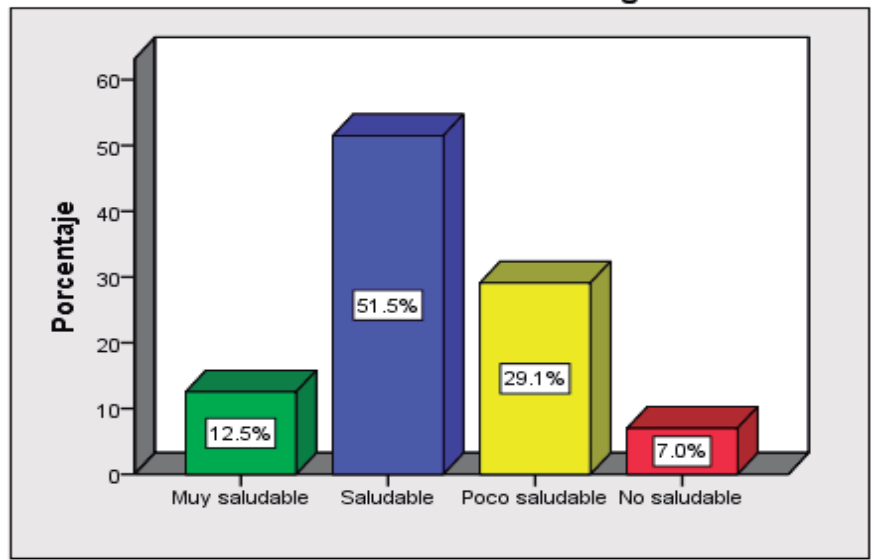

Figura 1. Representación gráfica del nivel del estilo de vida de los estudiantes de una universidad de Huánuco; 2014.

También, se comprobó que el predictor social: tenencia de trabajo paralelo al estudio fue predictor del estilo de vida poco saludable de los estudiantes $\left(X^{2}=18,08\right.$; Gl, 3 y $\mathrm{p}=0.00)$; por lo que se tuvo que rechazar la hipótesis nula (tabla 3 ).

Por el contrario, el hecho de proceder de la zona urbana $\left(\mathrm{X}^{2}=1,00 ; \mathrm{Gl}, 3\right.$ y $\left.\mathrm{p}=0,80\right)$, la tenencia de algún compromiso conyugal $\left(\mathrm{X}^{2}=2,26\right.$; Gl, 3 y $\left.\mathrm{p}=0,44\right)$, el profesar alguna religión $\left(\mathrm{X}^{2}=2,86 ; \mathrm{Gl}, 3\right.$ y $\left.\mathrm{p}=0,41\right)$, y la condición de cursar más del VI ciclo de estudios $\left(X^{2}=1,10\right.$ Gl, 3 y p $=0,78)$, no fueron predictores de los estilos de vida saludables de la muestra de universitarios; por lo que se aceptó las hipótesis nulas en cada caso. 
Tabla 1. Relación entre el predictor biológico: condición de pertenecer al grupo adolescente y el estilo de vida de los estudiantes de una Universidad de Huánuco 2014.

\begin{tabular}{|c|c|c|c|c|c|c|c|c|c|c|c|}
\hline \multirow{3}{*}{$\begin{array}{l}\text { Predictor biológico: } \\
\text { condición de } \\
\text { pertenecer al grupo } \\
\text { de edad adolescente }\end{array}$} & \multicolumn{8}{|c|}{ Estilo de vida } & \multirow{3}{*}{$\mathbf{X}^{2}$} & \multirow{3}{*}{ GI } & \multirow{3}{*}{ p-valor } \\
\hline & \multicolumn{2}{|c|}{ Muy saludable } & \multicolumn{2}{|c|}{ Saludable } & \multicolumn{2}{|c|}{ Poco saludable } & \multicolumn{2}{|c|}{ No saludable } & & & \\
\hline & $\mathbf{F i}$ & $\%$ & $\mathbf{F i}$ & $\%$ & $\mathbf{F i}$ & $\%$ & $\mathbf{F i}$ & $\%$ & & & \\
\hline Sí & 8 & 2,3 & 29 & 8,4 & 31 & 9,0 & 11 & 3,2 & & & \\
\hline No & 35 & 10,2 & 148 & 43,0 & 69 & 20,1 & 13 & 3,8 & 15,54 & 3 & 0,00 \\
\hline Total & 43 & 12,5 & 177 & 51,4 & 100 & 29,1 & 24 & 7,0 & & & \\
\hline
\end{tabular}

Fuente. Cuestionario de los predictores biosocioculturales y escala de estilos de vida.

Tabla 2. Relación entre el predictor biológico: condición de pertenecer al género masculino y el estilo de vida de los estudiantes de una Universidad de Huánuco 2014.

\begin{tabular}{|c|c|c|c|c|c|c|c|c|c|c|c|}
\hline \multirow{3}{*}{$\begin{array}{l}\text { Predictor biológico: } \\
\text { condición de pertenecer } \\
\text { al género masculino }\end{array}$} & \multicolumn{8}{|c|}{ Estilo de vida } & \multirow{3}{*}{$\mathbf{X}^{2}$} & \multirow{3}{*}{ GL } & \multirow{3}{*}{ p-valor } \\
\hline & \multicolumn{2}{|c|}{ Muy saludable } & \multicolumn{2}{|c|}{ Saludable } & \multicolumn{2}{|c|}{ Poco saludable } & \multicolumn{2}{|c|}{ No saludable } & & & \\
\hline & $\mathbf{F i}$ & $\%$ & $\mathbf{F i}$ & $\%$ & $\mathbf{F i}$ & $\%$ & $\mathbf{F i}$ & $\%$ & & & \\
\hline Sí & 21 & 6,1 & 64 & 18,6 & 57 & 16,6 & 14 & 4,1 & & & \\
\hline No & 22 & 6,4 & 113 & 32,8 & 43 & 12,5 & 10 & 2,9 & 13,35 & 3 & 0,00 \\
\hline Total & 43 & 12,5 & 177 & 51,4 & 100 & 29,1 & 24 & 7,0 & & & \\
\hline
\end{tabular}

Fuente. Cuestionario de los predictores biosocioculturales y escala de estilos de vida.

Tabla 3. Relación entre el predictor social: tenencia de trabajo paralelo al estudio y el estilo de vida de los estudiantes de una Universidad de Huánuco 2014.

\begin{tabular}{cccccccccccc}
\hline $\begin{array}{c}\text { Predictor social: } \\
\text { tenencia de } \\
\text { trabajo paralelo } \\
\text { al estudio }\end{array}$ & Muy saludable & Fi & Saludable & Poco saludable & No saludable & $\mathbf{X}^{2}$ & GL & p-valor \\
\hline Sí & 13 & 3,8 & Fi & \% & Fi & \% & Fi & \% & & & \\
No & 30 & 8,7 & 131 & 38,1 & 52 & 15,1 & 11 & 3,2 & 18,08 & 3 & 0,00 \\
\hline Total & 43 & 12,5 & 177 & 51,4 & 100 & 29,1 & 24 & 7,0 & & \\
\hline
\end{tabular}

Fuente. Cuestionario de los predictores biosocioculturales y escala de estilos de vida.

\section{DISCUSIÓN}

El desarrollo de la presente investigación partió de la premisa de que en las últimas décadas, existe un amplio consenso entre académicos investigadores que el estado de salud-enfermedad está determinado por la conducta, el estado psicológico y el entorno donde se vive. Al respecto, Meda, citado por Ríos (12), asiente la existencia de relación entre la conducta de las personas y su estado de salud (10) Departamento del Putumayo. [Tesis presentado como requisito de Especialización en Gerencia de la Salud Pública]; por tanto, el estudiante universitario al interactuar en el ambiente universitario, modifica sus actitudes y rutinas, modificando sus estilos de vida. Por cierto, surgen actividades y hábitos que pueden afectar el estilo y la calidad de vida; ejemplo las nuevas formas de vestir, interactuar, de movilizarse y administrar su tiempo, su alimentación, entre otros (1).

Los hallazgos del estudio determinan correspondencia entre los predictores biosocioculturales: edad, género y trabajo paralelo al estudio, los que se apoyan en el enfoque teórico de la promoción de la salud, que sustenta que dichos predictores son expresados colectivamente mediante la construcción de estilos de vida diversos, localizados fundamentalmente, en tiempo, o en espacios intersticiales de la vida, conformados por: predictores biológicos (incluye la edad y el género); predictores 
sociales (se considera el lugar de procedencia, el estado civil y la ocupación); y predictores culturales (el grado académico y la religión a su vez). En tanto los predictores sociales y culturales tienen como característica común que son aprendidos $\mathrm{y}$, por tanto, algunos pueden ser modificables a lo largo de toda la vida (6).

Marriner, Raile, citado por Acosta, Rodríguez (13), señalan que existen factores biosocioculturales, que son predictores de las conductas de salud a través de los factores cognitivo - perceptual. Estos factores pueden ser demográficos: como la edad, el sexo, la raza étnica, la educación, la procedencia y el ingreso económico; estos ejercen influencia indirecta a través de su impacto en el establecimiento de conductas de salud. En la misma línea Rodríguez y Agulló, aportan respecto al tema de la estructuración de la edad, al considerar que las diferentes franjas de edad en que se delimita la juventud varían de forma considerable; por tal, constituye un proceso psicosociológico que se transforma histórica y culturalmente (14). Los jóvenes que cursan estudios universitarios forman un grupo diferenciado del resto de la juventud, no sólo por la franja de edad en la que están comprendidos, sino también, y entendiendo siempre esto como una generalización y asumiendo los riesgos que ello conlleva, por su estatus económico y social, así como por el itinerario formativo que están recorriendo (y el capital formativo que supone esta trayectoria), y porque integran un grupo con un estilo de vida propio, frente al resto de jóvenes. Independientemente de estos factores, y aunque no se cumpliesen o fueran infundados, sí es reconocido social y culturalmente que los estudiantes universitarios constituyen un grupo diferenciado.

Corroboran el análisis Jessor et al., citados por Hernando et al., afirmando que el periodo adolescente es uno de los más decisivos para la adquisición de estilos de vida saludables, en el que tiene lugar la consolidación de algunos comportamientos provenientes de la infancia así como la incorporación de otros nuevos adquiridos en sus contextos de socialización (15). Por ello, es importante la adolescencia para el establecimiento del estilo de vida, el cual tiene que ver con los importantes cambios psicológicos y contextuales que tienen lugar durante estos años, en los que chicos y chicas realizan sus primeras salidas y reuniones con sus pares, sin la presencia de sus padres y en los que van ganando autonomía para tomar algunas decisiones relacionadas con el ocio y el estilo de vida (actividades extraescolares, consumo de sustancias, prácticas sexuales, etc.) a adoptar. Estos momentos de transición ofrecen muchas oportunidades para el desarrollo personal, pero también son de gran vulnerabilidad y riesgo, puesto que se trata de una edad crítica para el ensayo y aprendizaje de hábitos perjudiciales para la salud.

Osorio, citado por Vega (16), expresa que el género, la edad y la clase social, son ejes estructurales que constituyen la identidad y marcan nuestras trayectorias vitales. Las combinaciones de estas categorías dan lugar a estilos de vida, expectativas sociales, posiciones que implican diferencias y desigualdades sociales, especialmente como sociedad, donde el mercado se erige como el principal referente de coordinación social.

Un tema de análisis también fue el predictor social: tener un trabajo paralelo al estudio relacionado con los estilos de vida poco saludables, esto lo confirman Mondoñedo y Reyna (17), en un estudio desarrollado en Chimbote (Perú), al explicar que aquellos estilos menos favorables se dan en estudiantes que trabajan, ellos no equilibran estudio con trabajo y además no cumplen las demás recomendaciones de las conductas saludables.

Finalmente, el presente estudio pone en evidencia la importancia de los predictores de los estilos de vida de un grupo vulnerable, como son los estudiantes universitarios, ya que la realización saludable o no de estos, puede traer consigo importantes problemas de salud, tanto físicas, como psicológicas. Los hallazgos de esta investigación, implican intervenciones para el ejercicio pleno de la promoción de la salud, adicionalmente permite la planificación de servicios y políticas de salud física y mental dentro de la institución universitaria, objeto de estudio hacia una cualificación del estilo y calidad de vida, del desarrollo humano y el empoderamiento del proyecto de vida personal de los universitarios (1).

\section{REFERENCIAS BIBLIOGRAFICAS}

1. Páez ML, Castaño JJ. Estilos de vida y salud en estudiantes de una facultad de Psicología. Rev. Psicología desde el Caribe. 2010; (25):155-78.

2. Acuña Y, Cortes R. Promocion de estilos de vida saludable área de salud de Esparza. Tesis. San José, Costa Rica: Instituto Centro Americano de Salud Pública; 2012. (Citado el 15 de febrero del 2016) Disponible en: http://biblioteca. icap.ac.cr/BLIVI/TESIS/2012/acuna_castro_yessika_ sa 2012.pdf.

3. Organización de las Naciones Unidas. Asamblea General Consejo Económico y Social. Ginebra: Organización de las Naciones Unidas; 2005.

4. Aranda D, Cruz M. Factores biopsicológigos y socioculturales como predictores de la conducta promotora de salud en los estudiantes de Enfermería de la Universidad Adventista de Chile. Rev Cient de Ciencias de la Salud. 2014; 7(1):37-45. 
5. Lucas R, Santos DA, Pinheiro A, Bianchini TM, Luiz E. Determinantes sociodemográficos del estilo de vida en universitarios. Rev Salud Pública. 2012;16(3):382-93.

6. Cotrina JM, Rodríguez Y. Estilo de vida y factores biosocioculturales del adulto joven H.U.P. Lomas del Sur Nuevo Chimbote. Rev In Crescendo. 2014;5(1):13-22.

7. Gamarra ME, Rivera HS, Alcalde ME, Cabellos D. Estilo de vida, autoestima y apoyo social en estudiantes de enfermería. Rev. UCV-Scientia. 2010;2(2):73-81.

8. Ruiz M, Arce L, Ambicho A, Casimiro Z, Bueno M. Estilos de vida y estado nutricional de los estudiantes de la Universidad de Huánuco, 2014. Huanuco: Universidad de Huánuco; 2014.

9. Daza YD. Creencias y practicas sobre estilos de vida de los estudiantes de la escuela académico profesional de enfermería de la Universidad de Huánuco-2014. Tesis de grado Licenciada en Enfermería. Huánuco, Perú: Escuela Académico Profesional de Enfemería, Universidad de Huánuco; 2014.

10. Cerón EM, Muñoz FA, Oviedo MC, Rosero YA. Promoción de estilos de vida saludable en la zona urbana de la cabecera municipal de Mocoa, Departamento del Putumayo. Tesis presentado como requisito de Especialización en Gerencia de la Salud Pública. Putumayo: Convenio Universidad CES, Universidad Mariana; 2010.

11. Arrivillaga M, Salazar I, Correa D. Creencias sobre la salud y su relación con las prácticas de riesgo o de protección en jóvenes universitarios. Rev Colombia Médica. 2003;34(4):186-95.
12. Ríos MR. Estilo de vida y obesidad en estudiantes universitarios: una mirada con perspectiva de género. Alternativas en Psicología Rev Semestral. 2015(Número Especial):87-100.

13. Acosta G, Rodríguez Y. Estilo de vida y factores biosocioculturales de las madres de escolares del nivel primario de la I.E. César Vallejo - Nuevo Chimbote, 2012. (Citado el 15 de febrero del 2016) Disponible en: http://erp.uladech.edu.pe/archivos/03/03012/documentos/ repositorio/2013/01/12/021563/20140113121052.pdf.

14. Rodríguez J, Agulló E. Estilos de vida, cultura, ocio y tiempo libre de los estudiantes universitarios. Rev Psicothema. 1999;11(2):247-59.

15. Hernando Á, Oliva A, Pertegal ÁM. Diferencias de género en los estilos de vida de los adolescentes. Rev. Psychosocial Intervention. 2013;22(1):15-23.

16. Vega DI. Los discursos sobre la calidad de vida de hombres y mujeres mayores, desde una perspectiva de género. Tesis para optar al grado de Magíster en Estudios de Género y Cultura mención Ciencias Sociales. Santiago, Chile: Facultad de Ciencias Sociales, Universidad de Chile.; 2014.

17. Mondoñedo R, Reyna E. Estilo de vida y factores biosocioculturales de los adultos jóvenes. AA.HH. Carlos Garcia Ronceros - Nuevo Chimbote, 2011. Chimbote, Perú: ULADECH.

\section{Correspondencia:}

Mely Meleni Ruiz Aquino

Correo electrónico: melyruizaquino@hotmail.com

Fecha de recepción: 01 de julio del 2016.

Fecha de aceptación: 30 de noviembre del 2016. 